\title{
Is a SUV cut-off necessary in the evaluation of the response of axillary lymph node metastases to neoadjuvant therapy?
}

\author{
Laura Gilardi • Giovanni Paganelli
}

Received: 15 July 2010 / Accepted: 18 July 2010 /Published online: 7 September 2010

(C) Springer-Verlag 2010

Dear Sir,

We read with great interest the article by Straver et al. published in the June 2010 issue concerning the feasibility of FDG PET/CT for monitoring the response of axillary lymph node metastases to neoadjuvant chemotherapy in patients with breast cancer [1]. The authors submitted 38 patients to FDG PET/CT in order to determine how often the visualization of lymph node metastases is sufficiently prominent to allow monitoring of the axillary response to chemotherapy, and they defined a maximum standardized uptake value $\left(\mathrm{SUV}_{\max }\right)$ of $\geq 2.5$ as suitable for this purpose. We agree with the authors that it is crucial to have a sufficiently high baseline $\mathrm{SUV}_{\max }$ to monitor the early response of axillary lymph node metastases to therapy, since a low value could lead to underestimation of the subsequent tumour response. However, this could be of great value if it leads to changing an ineffective therapeutic regimen, which is not currently performed for the primary tumour and least of all for axillary metastases [2].

We believe that the response to treatment of axillary metastases should be assessed by PET/CT at the end of neoadjuvant therapy with the aim of determining the subsequent surgical treatment. This evaluation should be carried out in a binary way, evaluating the PET scan as either negative (complete disappearance of uptake in axillary lymph nodes) or positive (persistence of uptake in

\section{Gilardi · G. Paganelli $(\bowtie)$}

Division of Nuclear Medicine, European Institute of Oncology,

Via Ripamonti 435,

20141 Milano, Italy

e-mail: divisione.medicinanucleare@ieo.it axillary lymph nodes). If the PET scan is negative after neoadjuvant therapy, a sentinel node biopsy may be performed in order to spare the axilla if there is no metastatic involvement. Indeed, in this setting several studies have demonstrated the feasibility and accuracy of sentinel node biopsy, the most representative being the NSABP B-27 trial [3].

If there is persistent uptake at the axilla, there is no clear evidence to recommend the implementation of direct axillary dissection, although in a recent study we demonstrated high specificity and positive predictive value of PET in this setting [4].

Conflicts of interest None.

\section{References}

1. Straver ME, Aukema TS, Valdes Olmos RA, Rutgers EJ, Gilhuijs $\mathrm{KG}$, Schot ME, et al. Feasibility of FDG PET/CT to monitor the response of axillary lymph node metastases to neoadjuvant chemotherapy in breast cancer patients. Eur J Nucl Med Mol Imaging 2010;37:1069-76.

2. Avril N, Sassen S, Roylance R. Response to therapy in breast cancer. J Nucl Med 2009;50:55S-63S.

3. Mamounas EP, Brown A, Anderson S, Smith R, Julian T, Miller T, et al. Sentinel node biopsy after neoadjuvant chemotherapy in breast cancer: results from national surgical adjuvant breast and bowel project protocol B-27. J Clin Oncol 2005;23:2694-702.

4. Gilardi L, De Cicco C, Colleoni M, Cardillo A, Montagna E, Dellapasqua S, et al. Investigation of (18)F-FDG PET in the selection of patients with breast cancer as candidates for sentinel node biopsy after neoadjuvant therapy. Eur J Nucl Med Mol Imaging 2010. doi:10.1007/s00259-010-1494-1. 